RESEARCH ARTICLE

\title{
Healthcare Workers' Exposures for Mumps, Measles, Rubella and Varicella Zoster Virus Infections: A Multicenter Survey Study
}

\author{
Duygu Mert ${ }^{1}$, Selma Tosun ${ }^{2}$, Ilknur Esen Yildiz ${ }^{3}$, Handan Alay ${ }^{4}$, Duru Mistanoglu Ozatay ${ }^{5}$ Adult Vaccine \\ Working Group* \\ ${ }^{1}$ Department of Infectious Diseases and Clinical Microbiology, Ankara Oncology Education and Research Hospital, \\ Ankara, Turkey \\ ${ }^{2}$ Department of Infectious Diseases and Clinical Microbiology, University of Health Sciences, Bozyaka Training and \\ Research Hospital, Izmir, Turkey \\ ${ }^{3}$ Department of Infectious Diseases and Clinical Microbiology, Rize University Faculty of Medicine, Rize, Turkey \\ ${ }^{4}$ Department of Infectious Diseases and Clinical Microbiology, Nenehatun Obstetrics and Gynecology Hospital, \\ Erzurum, Turkey \\ ${ }^{5}$ Department of Infectious Diseases and Clinical Microbiology, Dumlupınar University Faculty of Medicine, Kutahya,
} Turkey

\begin{abstract}
Objectives: In this research article, the authors carried out a multi-center study to evaluate the exposure and vaccination status of selected Turkish health-care workers for measles, rubella, mumps, and varicella zoster.

Methods: The authors conducted a survey research by a questionnaire for health-care workers. The participants were questioned about vaccination and exposure of mumps, measles, rubella and varicella zoster virus.

Results: A total of 5165 health-care workers; 1665 male (32.2\%) and 3500 female $(67.8 \%)$ participated in the study. The rates of measles, rubella, mumps and varicella history in the participants were found to be $39.2 \%, 21.8 \%, 50.8 \%$ and $56.9 \%$, respectively. When health-care workers were asked about vaccination against measles, mumps and rubella; 2327 (47.4\%) participants could not remember, $1423(29 \%)$ participants stated that they had not been vaccinated and $763(15.5 \%)$ had been vaccinated. Only $55(1.1 \%)$ of the participants stated that they had measles vaccination. In total 2376 (49\%) participants stated that they had not been vaccinated against varicella zoster and 451 (9.4\%) people did not have vaccination since they already had developed disease and $1913(39.8 \%)$ people did not remember their vaccination status for varicella zoster. Only $68(1.4 \%)$ health-care workers stated that they had been vaccination against varicella zoster.
\end{abstract}

Conclusions: The authors found that only a small group of health-care workers were vaccinated against these diseases and most of them could not remember whether they had been exposed to these viruses. $J$ Microbiol Infect Dis 2019; 9(3):104-111.

Keywords: Measles, rubella, mumps and varicella zoster virus infections, vaccination

\section{INTRODUCTION}

Measles, rubella, mumps, and varicella infections are infectious diseases that are highly contagious. And it is possible to be protected by vaccination. These diseases spread from an infected person to a susceptible person through air-droplets. In addition, varicella infection can also be transmitted with contact of the fluid on the skin lesions [1]. These diseases are infectious and they might also be a risk for health-care workers. Although they could be prevented by vaccination and the incidence has been decreasing across the world, they still continue to occur both in our country and in the

\footnotetext{
*(Kenan Ugurlu, Mustafa Dogan, Busra Ergut Sezer, Merve Sefa Sayar, Ayhanim Tumturk, Secil Deniz, Zehra Karacaer, Mustafa Uguz, Farnaz Majdiehfarshi, Gul Durmus, Fatma Unlu, Yesim Uygun Kizmaz, Sirin Menekse, Nefise Oztoprak, Cagla Karakoc, Nuran Sari, Melike Betul Ogutmen, Cumhur Artuk, Emre Guven, Yasemin Balkan, Abdulkadir Daldal, Ayse Ozlem Mete, Meltem Tasbakan, Tansu Yamazhan, Deniz Akyol, Pinar Ergen, Ozlem Aydin, Selda Sayın, Rıza Aytac Cetinkaya, Ercan Yenilmez, Nurgul Ceran, Serpil Erol, Sinan Ozturk, Ayten Kadanalı, Arzu Altuncekic Yıldırım, Osman Ekinci, Ozgur Dagli, Hulya Ozkan Ozdemir, Ayse Batirel, Ergenekon Karagoz, Ugur Kostakoglu, Fazilet Duygu)
}

Correspondence: Dr. Duygu Mert, Department of Infectious Diseases, Ankara Oncology Education and Research Hospital, Ankara, Turkey 
world [2]. The most important factor in protection is vaccination in childhood since these diseases have more severe course in the adult age group. Adult immunization in our country is a newly spreading concept but it has not yet been at the desired level. Health-care workers are at higher risk of infection than individuals in the community and can also be a source of spread for these infections when they are infected [3]. When health-care workers suffer from the measles, rubella, mumps and varicella zoster infections, both themselves and their patients might be affected. Not every hospital in our country had all serological tests to determine whether these infectious agents had been encountered previously. For this reason, information about the infectious diseases can be obtained from the memory of the health-care workers and their families [4].

It is important to know the vaccination status of health-care workers about these communicable diseases that can be prevented by vaccination. In our study, we aimed to evaluate vaccination status of health-care workers on measles, rubella, mumps, and varicella diseases.

\section{METHODS}

Ethical approval was obtained from Clinical Research Ethics Committee of University of Health Sciences Bozyaka Training and Research Hospital (Approval date: 12/24//2013, number: 5). Informed consent was obtained from participants and all of the participants voluntarily participated the questionnaire study.

Table 1. Questions in the questionnaire.

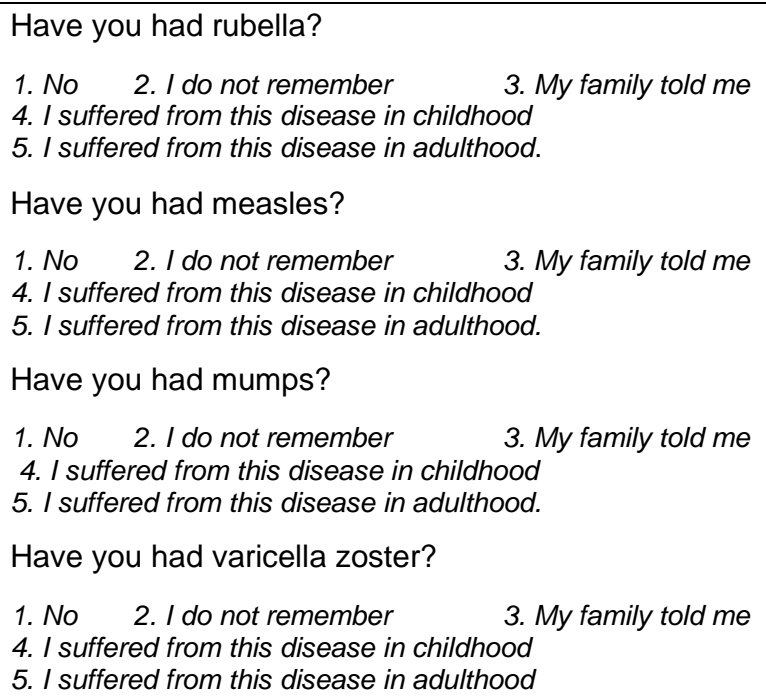

3. My family told me questionnaire are shown in Table 1. college level.

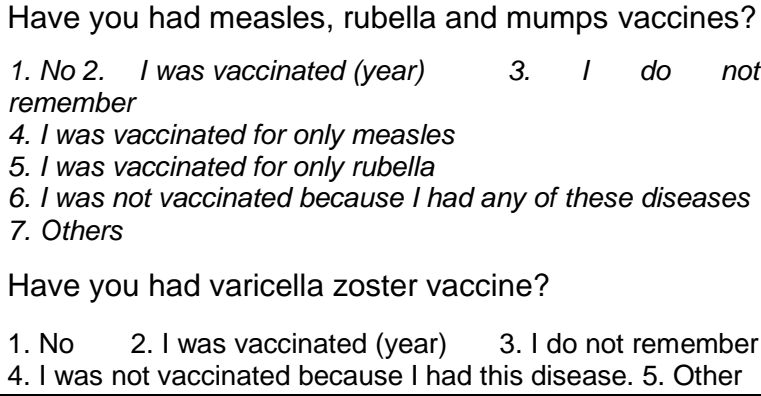

Following approval of the ethics committee and the approval of the Turkish Public Health Authority, the study was carried out as a multicenter study. This study was conducted in the various state hospitals and university hospitals in Turkey.

In the study, survey questions were asked to determine the socio-demographic data of healthcare workers and also to evaluate the transmission and immunization status of measles, rubella, mumps and varicella diseases.

The volunteer health-care workers were included in this study. Those who did not answer all of the questions or those who did not complete the questionnaire were excluded.

A web-based questionnaire was presented to the health-care workers and the study was conducted on a volunteer basis. All the data of this research article were obtained from the study subjects.

\section{RESULTS}

A total of 5165 health-care workers, 1665 $(32.2 \%)$ male and 3500 (67.8\%) female participated in the study and the age of participants were between 16 and 67 years old. The institutions of the participants in

In terms of educational status; the majority of the participants were educated at university and

In terms of profession groups; $1140(22 \%)$ of participants were medical doctors, 254 (4.9\%) were medical faculty students, 1909 (36.9\%) were nurses, 409 (7.9\%) were laboratory workers and $320(6.2 \%)$ were employees of cleaning companies, $513(9.9 \%)$ were hospital office workers, $317(6.1 \%)$ were trainee students and $303(5.9 \%)$ were other health-care workers. 
The professions of the participants are shown in Table 2.

According to the statements of health-care workers, their history of transmitted measles, rubella, mumps and varicella infections and vaccination statuses are shown in Table 3.

In this study, the percentage of participants who never had measles, rubella, mumps and varicella zoster diseases were $28.3 \%, 36.8 \%$, $25.7 \%$ and $\% 17.8$ respectively. The percentage of those who did not remember having measles, rubella, mumps and varicella diseases were $32.4 \%, 41.4 \%, 23.4 \%$ and $25.3 \%$ respectively. The percentage of participants with the history of measles, rubella, mumps and varicella were found to be $39.2 \%, 21.8 \%, 50.8 \%$ and $56.9 \%$ respectively. They remembered their history of measles, rubella, mumps and varicella. It was easy to remember because these infections could cause rash.

Table 1. The institution of participants in the study.

\begin{tabular}{lc}
\hline Institution & $\begin{array}{c}\text { Number (\%) of } \\
\text { participants }\end{array}$ \\
\hline Family health center & $153(2.9 \%)$ \\
Community health center & $59(1.1 \%)$ \\
State hospital & $1493(28.9 \%)$ \\
Training and research & $2720(52.7 \%)$ \\
hospital & $565(11 \%)$ \\
University hospital & $127(2.5 \%)$ \\
Private hospital & $23(0.4 \%)$ \\
Private university hospital & $6(0.1 \%)$ \\
Consulting room & $15(0.3 \%)$ \\
112 emergency & $1(0.01 \%)$ \\
Pharmacy &
\end{tabular}

\begin{tabular}{lc}
\hline Institution doctor & $1(0.01 \%)$ \\
Other institutions & $2(0.03 \%)$ \\
Total & $5165(100 \%)$ \\
\hline
\end{tabular}

When health-care workers were asked about their vaccination history against measles, mumps and rubella; 763 (15.5\%) of the participants had measles, mumps and rubella vaccination and 55 (1.1\%) participants had vaccination only against measles.

In this study, we found that 2376 (49.0\%) participants were never vaccinated, 451 (9.4\%) people were not vaccinated since they already had that disease and 1913 (39.8\%) people did not remember their vaccination status for varicella zoster. As a result, only 68 (1.4\%) health-care workers were vaccinated for varicella zoster.

Table 2. Occupation of participants.

\begin{tabular}{lc}
\hline Occupation & $\begin{array}{c}\text { Number (\%) of } \\
\text { participants } \\
\text { Medical doctor }\end{array}$ \\
Medical faculty student & $254(4.9 \%)$ \\
Dentist, pharmacist & $40(0.8 \%)$ \\
Nurse, midwife & $1909(36.9 \%)$ \\
Laboratory workers & $409(7.9 \%)$ \\
Employees of cleaning & $320(6.2 \%)$ \\
companies & \\
Transportation, security, & $148(2.8 \%)$ \\
kitchen workers & \\
Office workers & $513(9.9 \%)$ \\
Trainee students & $317(6.1 \%)$ \\
Others & $115(2.3 \%)$ \\
Total & $5165(100 \%)$
\end{tabular}

Table 3. Self-reported disease history in healthcare workers.

\begin{tabular}{lccccc}
\hline $\begin{array}{l}\text { Disease } \\
\text { name }\end{array}$ & $\begin{array}{c}\text { Number of } \\
\text { participants }\end{array}$ & $\begin{array}{c}\text { Number with no } \\
\text { disease history }\end{array}$ & $\begin{array}{c}\text { Number with no } \\
\text { memory of disease }\end{array}$ & $\begin{array}{c}\text { Number with the } \\
\text { disease history }\end{array}$ & $\begin{array}{c}\text { Vaccination } \\
\text { status }\end{array}$ \\
\hline Rubella & 5011 & $1843(36.8 \%)$ & $2076(41.4 \%)$ & $1092(21.8 \%)$ & $763(15.5 \%)$ \\
Measles & 5011 & $1418(28.3 \%)$ & $1625(32.4 \%)$ & $1968(39.2 \%)$ & $818(16.6 \%)$ \\
Mumps & 5008 & $1287(25.7 \%)$ & $1171(23.4 \%)$ & $2550(50.8 \%)$ & $763(15.5 \%)$ \\
Varicella & 5004 & $893(17.8 \%)$ & $1266(25.3 \%)$ & $2845(56.9 \%)$ & $68(1.4 \%)$ \\
zoster & & & & & \\
\hline
\end{tabular}




\section{DISCUSSION}

In the study of Zencir et al., it was reported that history of varicella zoster $(58.0 \%)$ and mumps (56\%) were found at a high degree and since the vaccination was performed in childhood this status was mostly reported by their families [4]. In the study done by Cilız et al, according to the disease history of the participants at the higher rates they remembered diseases that varicella zoster $(56.6 \%)$, mumps $(47.9 \%)$ and measles $(34.3 \%)$ [5]. Also, in the study of Sengoz et al., according to the disease history of the participants, they mostly remembered mumps $(61.7 \%)$, measles $(59.6 \%)$ and varicella zoster (59.1\%) [6]. Güner performed a study at an education and research hospital in 2016, with the participation of 491 health-care workers with a mean age of 30.5 years old, the participants were interviewed for measles, rubella, mumps, and varicella zoster infections. According to the result the most commonly reported infections were varicella zoster $(60.1 \%)$, mumps $(47.0 \%)$, measles (37.9\%) and rubella (17.8\%) [7]. It was obvious that recalling infectious agents such as varicella and mumps are more frequent than diseases with rashes such as measles and rubella [7]. Therefore, in this study, it was determined that varicella (38.3\%) and mumps (\%36.3) were the most commonly remembered infectious diseases among health-care workers. In addition, varicella (17.3\%) and mumps $(13.5 \%)$ were the most common reported diseases by their families. The high recall rates of these two diseases might be due to the fact that the clinical findings are clearer than the other diseases. Also, in our study, varicella $(17.3 \%)$ and measles (16\%) infections were similarly the most reported diseases by the families of the participants.

In another study conducted by Tosun et al., in 178 health-care worker participants, $10.4 \%$ of health-care workers reported that their family had told them they suffered from the mumps disease and $55 \%$ of health-care workers remembered that they had suffered from the mumps in childhood. $17.6 \%$ of health-care workers reported that their families had told them that they had suffered from varicella disease, and $57 \%$ of health-care workers remembered that they had suffered from varicella disease in their childhood [8]. In the study of Sengoz et al., 71 of the participants $(18.5 \%)$ stated that they had measles, mumps and rubella vaccination and 28 of the participants $(7.3 \%)$ had varicella vaccination [6]. In the study of Ziegler et al. 35\% of the healthcare personals could not remember the history of measles, mumps and rubella vaccination and $23 \%$ of the health-care personals could not remember whether they had suffered from measles disease [9]. According to this study, it was observed that the vaccination of measles, mumps and rubella was not so much.

In a study conducted by Alp et al., in 1255 health-care workers, positive predictive values of history of measles, rubella, mumps vaccinations and varicella vaccination were found to be $96 \%$, $100 \%, 93 \%$ and $98 \%$, respectively and negative predictive values of history of measles, rubella, mumps vaccination and varicella vaccination were found to be $13 \%, 5 \%, 17 \%$ and $2 \%$, respectively [10]. Zencir et al., found the rates of vaccination of health-care workers as $90 \%$ for mumps, $94 \%$ for measles, $97 \%$ for rubella and $98 \%$ for varicella [4]. In the study of Celikbas et al.; positive predictive value of measles, mumps and rubella history was found to be $92 \%$, and positive predictive value of varicella history was found as $100 \%$ in 363 health-care workers [11].

In a multicenter study performed with 436 participating health-care workers in Italy, a positive predictive value for the history of disease transmission found as $95 \%$ for the measles, rubella, mumps and varicella infections [12]. Additionally, in the study of Sengoz et al. the positive predictive values of the disease history of the participating health-care workers for the measles, rubella, mumps and the varicella found as $95.6 \%, 98.9 \%, 97.5 \%$ and $96.9 \%$, respectively [6].

In various studies it has been found that it is not cost-effective to vaccinate health-care workers with measles, mumps and rubella vaccine without knowing their vaccination status $[13,14]$. In the study of Ciliz et al. $308(99.7 \%)$ of the health-care workers were found to be positive for antibodies against measles, mumps and varicella and 299 (96.8\%) of the health-care workers were found to be positive for antibodies against rubella [5]. In the study of Sengoz et al. rubella, mumps and varicella zoster 
seropositivity were found to have $92.2 \%, 98.2 \%$, $94 \%$ and $94.3 \%$ respectively in health-care workers [6]. In the study done by Gulec et al., seropositivity of varicella was found as $97.4 \%$ in health-care workers [15]. In a study performed at a tertiary hospital in Italy, the rates of vaccination of 333 health-care workers were found as $98.2 \%$ for measles, $97.6 \%$ for rubella, $85.9 \%$ for mumps and $97.9 \%$ for varicella zoster [16].

Despite the low rate of disease and vaccination, the high seropositivity rate has been attributed to the fact that the majority of diseases were subclinical and naturally transmitted, or that vaccination and illness were not remembered [5]. In a study done in Israel, seropositivity of varicella was found to be $98.5 \%$ in 200 health employee [17]. In a study performed in Brazil, seropositivity of varicella was reported as $72.7 \%$ in 182 health-care worker [18]. While in a study in Italy, performed with 3600 health-care personals, seropositivity rate of varicella was found as $85.7 \%$ [12]. Also, in a study done in Spain, in 644 health-care workers seropositivity of varicella was found to be $94.9 \%$ [19].

In our study, 2376 (49\%) participants stated that they haven't been vaccinated for varicella zoster and $451(9.4 \%)$ participants stated that they were not vaccinated because they had suffered from varicella zoster disease in their childhood. $1913(39.8 \%)$ participants stated that they did not remember their status of varicella vaccination. Besides, only 68 (1.4\%) health-care workers stated that they were vaccinated for varicella infection.

This study is a multicenter study performed to determine the prevalence of four childhood diseases and their vaccination status of healthcare workers. The results of the study showed that only a small group of health-care workers have been vaccinated against measles, rubella, mumps, and varicella. And most of the healthcare workers did not remember whether they had suffered from these diseases. It has been observed that a large group of health-care workers did not have vaccination since they suffered from these diseases in their childhood. On the other hand, it is observed that healthcare workers cared about hepatitis $B$ vaccination and a large group of health-care workers were vaccinated against hepatitis $B$. However, we observed that a small group of health-care workers were vaccinated against measles, mumps and rubella and varicella.

Infectious diseases are important for all ages and adults should be vaccinated against these diseases. Immunization should continue for life. Therefore vaccination is also important for adults over 18 years [20]. Especially immunization is plays an important role in prevention of vaccinepreventable diseases and death in the elderly. Immunization can reduce the incidence of many diseases and is cost-effective [21]. Today, adult vaccination is still not at ideal levels. Adult vaccination rates are very low in our country and adult vaccination rates of $2 \%$ are below in our country [21].

The serological tests should be used to control the immunological statuses of health-care personals working at risky departments. Formal documentation of immunity to measles, mumps, and rubella should be recorded for health-care workers [22]. Health-care workers should be screened for varicella zoster disease serological tests at the time of initial employment; those without evidence of immunity should be immunized if there are no contraindications [23]. Evidence of immunity for health-care workers includes any of the following [23]: Written documentation of vaccination with two doses of varicella vaccine, laboratory evidence of seropositivity [23].

In conclusion, to determine their susceptibility to these vaccine-preventable diseases, health-care workers should be monitored with a seroprevalence survey and should be immunized with the necessary vaccination programs. Health-care workers should have their serological tests checked before starting work. There are adult vaccination programs in our country. Therefore, we believe that this kind of field surveys will be useful in determining the vaccination policies of the country. We believe that it is good to have for the necessary tests for vaccination before health-care workers start to the job.

In addition, conducting similar studies in each center will contribute to the determination of precontact and post-contact institutional strategies, as well as to the creation of screening and immunization programs for health-care workers in our country 


\section{ACKNOWLEDGMENTS}

We would like to thank other health-care workers for their contributions to the study.

Declaration of Conflicting Interests: The authors declare that they have no conflict of interest.

\section{Funding: None of declared. REFERENCES}

1. Centers for Disease Control and Prevention. Progress toward measles elimination-Japan, 19992008. MMWR 2008; 57(38):1049-52.

2. Guris D, Bayazit $Y$, Ozdemirer $U$, et al. Measles epidemiology and elimination strategies in Turkey. J Infect Dis 2003; 187 Suppl 1:S230-S234.

3. Centers for Disease Control and Prevention. Immunization of Health-care Workers: Recommendation of the Advisory Committee on Immunization Practices (ACIP) and the Hospital Infection Control Practices Advisory Committee (HICPAC). MMRW 1997; 46(RR-18):1-42.

4. Zencir M, Bal F, Guler M, et al. The evaluation of exposure status against the measles, mumps, rubella and the varicella zoster virus infections among the health care workers. Izmir Training and Research Hospital Medical Journal 2014; 18 (4): 108-112.

5. Ciliz N. Determination of measles, rubella, mumps, varicella, diphtheria, tetanus and HBV antibody levels in health care workers, Celal Bayar University Hospital, Department of Medical Microbiology, Thesis, Manisa 2013

6. Sengoz M. Seroprevalence of measles, rubella, mumps, varicella in health care workers. Bulent Ecevit University Hospital, Department of Infectious Diseases and Clinical Microbiology, Thesis, Zonguldak 2015.

7. Guner E. Determination of awareness and immunization status of diseases and vaccination that can be protected by the health care workers. University of Health Sciences, Bozyaka Training and Research Hospital, Department of Infectious Diseases and Clinical Microbiology, Thesis, Izmir 2017.

8. Tosun S, Deveci S, Kaplan Y, Isbir B, Yilmaz T. EKMUD Congress, 24-28 October, Ankara, 259, p170.

9. Ziegler E. Prevalence of measles susceptibility among health care workers in a UK Hospital. Does the UK need to introduce a measles policy for its health care workers? Occupat Med 2003; 53:398-440.

10. Alp E, Cevahir F, Gokahmetoglu S, Demiraslan H, Doganay M. Prevaccination screening of health care workers for immunity to measles, rubella, mumps, and varicella in a developing country: What do we save? J Infect Public Health 2012; 5: 127-132.
11. Celikbas A, Ergonul O, Aksaray S, et al. Measles rubella, mumps, and varicella seroprevalence among health care workers in Turkey: is prevaccination screening cost-effective? Am J Infect Control 2006; 34:583-587.

12. Taddei C, Ceccherini V, Niccolai G, et al. Attitude toward immunization and risk perception of measles, rubella, mumps, varicella and pertussis in health care workers in 6 hospitals of Florance, Italy 2011. Hum Vaccin Immunother 2014; 10: 2612-2622.

13. Giri P, Basu S, Farrow D, Adisesh A. Costeffectiveness analysis of MMR immunization in health care workers. Occup Med 2013; 63:422-4.

14. Chodick G, Ashkenazi S, Livni G, Lerman Y. Costeffectiveness of varicella vaccination of health care workers. Vaccine 2005; 17;23:5064-5072.

15. Gulec H. Varicella and hepatitis an antibody prevalance in health care workers. University of Istanbul, Istanbul Faculty of Medicine, Department of Medical Microbiology. Thesis, Istanbul 2013.

16. Fedeli U, Zanetti C, Saia B. Susceptibility of health care workers to measles, mumps, rubella and varicella. J Hosp Infect 2002; 51: 133-135.

17. Chazan B, Colodner R, Teitler N, Chen Y, Raz R. Varicella zoster virus in health care workers in Northern Israel: seroprevalence and predictive value of history of varicella infection. Am J Infect Control 2008; 36:436-438.

18. Dinelli MI, Moreira Td, Paulino ER, da Rocha MC, Graciani FB, de Moraes-Pinto MI. Immune status and risk perception of acquisition of vaccine preventable diseases among health care workers. Am J Infect Control 2009; 37: 858-860.

19. Borras E, Campins M, Working Group for the Study of the Immune Status in Health care Workers. Are health care workers immune to rubella? Hum Vaccin Immunother 2014; 10: 686-691.

20. Koksal I, Usluer G. Adult Immunization. ANKEM. 2006; 20(4):239-245.

21. National Vaccine Workshop Report. T. C. Ministry of Health Infectious Diseases Association. 27-29 March 2014, Ankara.

22. Kimberlin DW, Brady MT, Jackson MA, Long SS 2018-2021 Report of the Committee on Infectious Diseases Red Book, $31^{\text {st }}$ ed. Itasca, IL 2018.

23. Centers for Disease Control and Prevention: Immunization of health care personnel: recommendations of the Advisory Committee on Immunization Practices (ACIP). MMWR 2011; 60 (No. RR-7):1-45. 